\title{
Social Force Modeling of the Pedestrian Motion in the Mataf
}

\author{
Rainald Löhner ${ }^{1}$, Eberhard Haug ${ }^{2}$, Britto M. ${ }^{2}$ \\ ${ }^{1}$ Center for Computational Fluid Dynamics \\ George Mason University, Fairfax, VA, USA \\ rlohner@gmu.edu \\ ${ }^{2}$ SL Rasch \\ Kesslerweg 22, Oberaichen, Germany \\ britto.muhamad@sl-rasch.de, haugfmly@club-internet.fr
}

\begin{abstract}
Two models for the motion in the Mataf of the Holy Mosque of Makkah that are applicable to any so-called social force or agent-based model are presented. The first model assigns a desired distance to the Kaaba to each pilgrim. The second model assumes that pilgrims desire to get as close as possible to the Kaaba until a tolerable density is reached. Both models are realistic: image data shows behaviours of both types. The models are very simple to incorporate into any computational crowd dynamics code, and yield results that correlate surprisingly well with observed data.
\end{abstract}

Keywords: Pedestrian Modeling, Social Force Model, Crowd Dynamics, Kaaba, Hajj

\section{Introduction}

One of the obligatory rites of Muslim pilgrims visiting Makkah is the sevenfold circumambulation of the Kaaba at the center of the Holy Mosque - the so-called Mataf. During the peak holy seasons of Ramadan and Hajj millions of pedestrians perform this rite, leading to congestion and, in some cases, worrysome densities. The motion of pedestrians in the Mataf has been the focus of much modeling effort over the last years. Unfortunately, at this point there does not exist a reliable predictive model for this motion. This should not come as a surprise: after all, at every instance a pilgrim is taking decisions based on many factors: faith, social and cultural background, current animic and fitness state, the surrounding crowd, climate, etc. Following the observation that 'all models are wrong, but some are useful', the present effort was directed at developing improved social force models for this type of motion/rite. Starting from a series of empirically based assumptions, two models were derived in a systematic manner. The first model assumes that pilgrims want to perform the Tawaf at a certain distance from the Kaaba. Video footage shows that even when there are very few pilgrims, some pilgrims do not venture close to the Kaaba, implying that this model is not unrealistic. The second model assumes that pilgrims want to get as close as possible to the Kaaba when performing the Tawaf but have a limited tolerance for high density. This implies that they will stop trying to get closer to the Kaaba if the density is above a certain threshold. Video footage shows that even when there are very many pilgrims, some pilgrims keep trying to get to the Kaaba or very close to it, i.e. are not afraid of high densities. 


\section{Model 1: Desired Distance from Kaaba}

The first model assumes that pilgrims want to perform the Tawaf at a certain distance from the Kaaba. Video footage shows that even when there are very few pilgrims, some pilgrims do not venture close to the Kaaba, implying that this model is not unrealistic. The desire to stay at a certain distance from the Kaaba is modeled by assigning to each pilgrim a 'desired distance' $\boldsymbol{d}_{\boldsymbol{d}}$. The inward or outward force is then a function of the difference in desired distance $\boldsymbol{d}_{\boldsymbol{d}}$ and actual distance $\boldsymbol{d}$ from the Kaaba.

\section{Model 2: Tolerated Density}

The second model assumes that pilgrims want to get as close as possible to the Kaaba when performing the Tawaf but have a limited tolerance for high density. This implies that they will stop trying to get closer to the Kaaba if the density is above a certain threshold. Video footage shows that even when there are very many pilgrims, some pilgrims keep trying to get to the Kaaba or very close to it, i.e. are not afraid of high densities. The desire to stay in regions where pilgrim density is below a certain threshold is modeled by assigning to each pilgrim a 'threshold density' $\boldsymbol{\rho}_{0}$. The inward or outward force is then a function of the difference between the local density $\boldsymbol{\rho}$ and the threshold density $\boldsymbol{\rho}_{\boldsymbol{0}}$.

\section{Examples}

Both models were implemented into PEDFLOW [1-3], a typical pedestrian flow and crowd dynamics simulation tool based on a mix of social force and agent-based approaches. In order to discern the relative merit and performance of the two models, the Mataf motion was computed with the maximum number of simplifying assumptions that still reflect reality.

a) Geometry: The Great Mosque in Makkah has been undergoing construction for the last decade, so the areas where pilgrims can walk and pray are constantly changing. However, the geometry chosen closely reflects the actual dimension and areas expected in the next decade.

b) Entry/Exit Points: Pilgrims are assumed to enter through one entry into the central patio (the so-called Sahn) and exit through one area. From a series of exploratory simulations it was found that the crowd motion patterns that form are almost unaffected by the actual form of entry into and exit from the Sahn as long as there is enough space for pilgrims to move through.

c) Sunnah Prayer: A part of the actual pilgrimage rite that was left out was the post-Mataf prayer. Pilgrims remain in the general area of the Sahn and pray before exiting. As these prayer areas were not clearly defined for the geometry chosen, they were omitted.

d) Demographics: Only one demographic type was considered, with a desired velocity of $\mathrm{v}_{\mathrm{d}}=1.5+/-0.25 \mathrm{~m} / \mathrm{sec}$, a relaxation time of $\mathrm{t}=0.5+/-0.05 \mathrm{sec}$, and radius of $\mathrm{r}=0.25+/-0.05 \mathrm{~m}$.

e) Constant Influx: The influx of pedestrians was set to $\mathrm{f}=9 \mathrm{p} / \mathrm{sec}=32,400 \mathrm{p} / \mathrm{hr}$, constant for the duration of the simulation. This is considered typical of high holidays.

As stated before, more realism and complexity could easily have been added, but it would not have been helpful to elucidate the relative merits of the models proposed.

\subsection{Distance Preference}

The case was first run with the model based on a desired distance. Figure 1 shows the average density, average velocity, number of pedestrians and density vs distance from the Kaaba at $T=15,000 \mathrm{sec}$. Note that the velocities are only displayed in the range $0<|\mathrm{v}|<1$ in order to clarify the regions with slower motion. Note the unrealistically low average values for the density close the Kaaba obtained with the model, as well as the low density regions close to the left part of the Kaaba. Nevertheless, the 'flow pattern' is much better than that obtained with many other approaches we tried to date. 

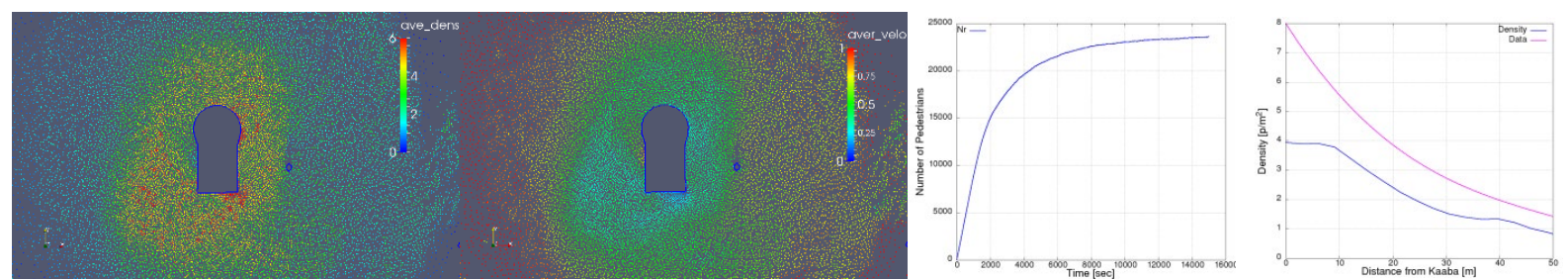

Figure 1: Average Density and Velocity, Number of Pedestrians and Density vs Distance from Kaaba

\subsection{Density Preference}

The same case was then run with the model based on a desired density. Figure 2 shows the average density, average velocity, number of pedestrians and density vs distance from the Kaaba at $\mathrm{T}=10,000 \mathrm{sec}$. As before, the velocities are only displayed in the range $0<|\mathrm{v}|<1$ in order to clarify the regions with slower motion. Note the high values of density close the the Kaaba obtained with the model, which correlate well with observed data.
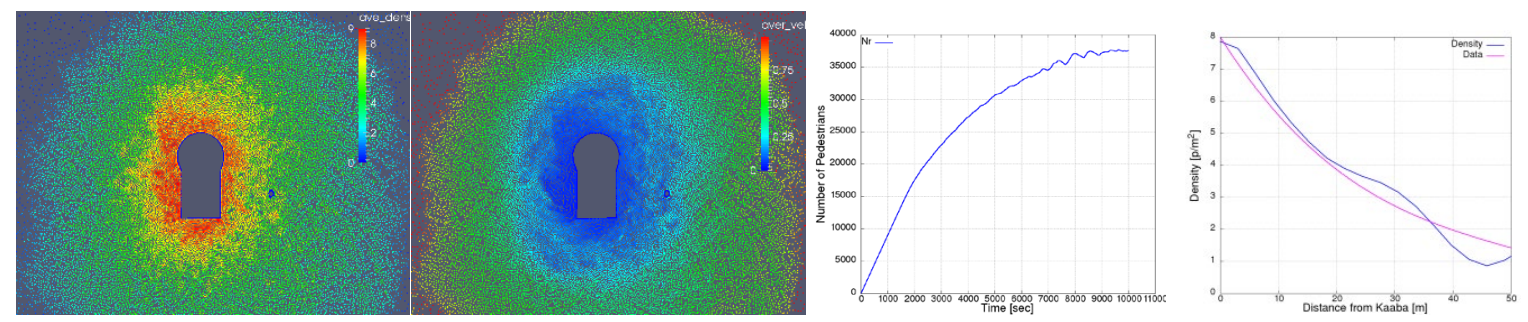

Figure 2: Average Density and Velocity, Number of Pedestrians and Density vs Distance from Kaaba

\section{Conclusions and Outlook}

Two models for the motion in the Mataf that are applicable to any so-called social force model were presented. The first model assigns a desired distance to the Kaaba to each pilgrim. The second model assumes that pilgrims desire to get as close as possible to the Kaaba until a tolerable density is reached. Both models are realistic: image data shows behaviours of both types. The second model offers the advantage of being self-regulating and thus more robust. If the density increases too much, the pilgrims move further away from the Kaaba and the simulation proceeds without problems while still being realistic. Both models are very simple to incorporate into any computational crowd dynamics code, and yield results that correlate well with observed data.

As in all such cases, it is tempting to overinterpret the results obtained. Both behaviours (desired distance, density threshold) are observed in the field. What percentage will opt for any of these goals remains an open question.

\section{References}

[1] R. Löhner, "On the Modeling of Pedestrian Motion," Appl. Math. Modelling 34, 2, 366-382 (2010).

[2] R. Löhner, E. Haug, C. Zinggerling and E. Onate, "Real-Time Micro-Modeling of City Evacuations," Comp. Part. Mech. 5, 71-86 (2018).

[3] R. Löhner, B. Muhamad, P. Dambalmath and E. Haug, "Fundamental Diagrams for Specific Very High Density Crowds," Collective Dynamics 2 (2018). 